\title{
Ultrafast dynamics of lattice relaxation of excitons in quasi-one-dimensional halogen-bridged platinum complexes
}

\author{
Shinichi Tomimoto, ${ }^{*}$ Shingo Saito, and Tohru Suemoto \\ The Institute for Solid State Physics, The University of Tokyo, Kashiwa, Chiba 277-8581, Japan \\ Jun Takeda and Susumu Kurita \\ Department of Physics, Faculty of Engineering, Yokohama National University, Yokohama 240-8501, Japan \\ (Received 18 March 2002; published 24 October 2002)
}

\begin{abstract}
The temporal evolution of the photoexcited state in quasi-one-dimensional (1D) halogen-bridged platinum complexes $\left[\mathrm{Pt}(\mathrm{en})_{2}\right]\left[\mathrm{Pt}(\mathrm{en})_{2} X_{2}\right]\left(\mathrm{ClO}_{4}\right)_{4}$ (abbreviated as $\mathrm{Pt}-X, X=\mathrm{Cl}, \mathrm{Br}$ or I), has been comprehensively studied by femtosecond time-resolved luminescence spectroscopy. In $\mathrm{Pt}-\mathrm{Cl}$, new short-lived hot luminescence is found in the low-energy side of a self-trapped exciton (STE) luminescence band. The overall behavior of the STE luminescence band within 2 ps is well explained by the vibrational relaxation of the STE. The behavior is reproduced by a model calculation based on wave packet propagation on an interaction mode composed of frequency-dispersed bulk phonons. This model is also applied to the previous results in $\mathrm{Pt}-\mathrm{Br}$. For both $\mathrm{Pt}-\mathrm{Cl}$ and Pt-Br, the frequency spectra of phonons which compose the interaction mode have been estimated. In Pt-I, the STE luminescence decays much faster than those in $\mathrm{Pt}-\mathrm{Cl}$ and $\mathrm{Pt}-\mathrm{Br}$, showing existence of more effective nonradiative decay channel.
\end{abstract}

DOI: 10.1103/PhysRevB.66.155112

PACS number(s): 71.35.Aa, 78.47.+p, 78.55.Hx

\section{INTRODUCTION}

Structural changes induced by electronic excitation have been of continued interest in condensed matter physics. It is well known that the photo-generated free carriers and excitons in insulators often bring about the local lattice deformation and become localized. Such self-trapping phenomena have been studied over years, ${ }^{1}$ However, from the experimental point of view, some basic problems are still left open. One of them is how the localization of electronic wave function is triggered and what kind of phonon mode stabilizes the localized state. We can observe the so-called local mode (or defect mode) relevant to the localized electrons by Raman and far-infrared (FIR) spectroscopy. It is often assumed implicitly that this local mode triggers the localization and stabilizes energetically the localized electronic state. However, there is no obvious experimental evidence that shows this special normal mode is responsible for triggering the localization and for energetic stabilization.

It gives valuable information on the self-trapping mode to observe directly the temporal evolution of a system during the self-trapping process by femtosecond spectroscopic experiments. In particular, the time-resolved luminescence measurement is suited for this purpose. This is because the wave packet sliding along the mode can be observed at arbitrary points simply by varying the detection photon energy. The correspondence of the photon energy with the configuration coordinate along the mode is very simple because it depends only on the potential energy curves of the ground and the first excited states.

Halogen-bridged platinum complexes $\left[\mathrm{Pt}(\mathrm{en})_{2}\right]$ $\left[\mathrm{Pt}(\mathrm{en})_{2} \mathrm{X}_{2}\right]\left(\mathrm{ClO}_{4}\right)_{4}$ (hereafter, these are abbreviated as $\mathrm{Pt}-X$, en $=$ ethylenediamine. $X=\mathrm{Cl}, \mathrm{Br}$, or I.) are typical quasi-one-dimensional (1D) strongly coupled electron-lattice systems. Their crystal structure is characterized by a linear chain composed of alternating $\mathrm{Pt}$ and halogen ions. This chain can be expressed as the sequence $\cdots-\mathrm{Pt}^{3-\rho}-X^{-}$ $-\mathrm{Pt}^{3+\rho}-X^{-}-\cdots$, where $\rho(0 \leqslant \rho \leqslant 1)$ represents the deviation of the valency of platinum ions from 3 . The halogen ions on the chain are located closer to $\mathrm{Pt}^{3+\rho}$ than to $\mathrm{Pt}^{3-\rho}$. This mixed-valence state is understood as a commensurate charge-density wave (CDW) caused by the Peierls instability of 1D metal. In Table I, the fundamental structural and optical properties of Pt- $X$ are summarized. The ratio of Pt- $X$ distances $d_{2} / d_{1}$ becomes smaller with increasing the halogen ion radius because it is more difficult for larger ions to approach $\mathrm{Pt}^{3+\rho}$ ions. The single crystals of $\mathrm{Pt}-X$ are known to exhibit an intense chain-axis-polarized optical absorption band from near-infrared to visible wavelength range. ${ }^{2}$ This absorption band has been assigned to a charge-transfer (CT) optical excitation of an electron from $\mathrm{Pt}^{3-\rho}$ to $\mathrm{Pt}^{3+\rho}$. It gives

TABLE I. Fundamental structural and optical properties of Pt- $X . d_{1}$ and $d_{2}$ are distances of $\mathrm{Pt}^{3+\rho}-X^{-}$and $\mathrm{Pt}^{3-\rho}-X^{-}$, respectively (Refs. 3, and 4). $\rho$ represents the deviation in valency of Pt ions from 3 (Ref. 2). $E_{\mathrm{CT}}$ and $E_{\mathrm{lm}}$ are the peak energies of the CT exciton absorption band at room temperature and the STE luminescence band at $2 \mathrm{~K}$, respectively (Ref. 2). $\omega_{\mathrm{R}}$ is the frequency of the Raman-active symmetric stretching mode (Refs. 5-7). $T_{\text {vib }}$ is the vibration period of the mode.

\begin{tabular}{lccc}
\hline \hline Pt- $X$ & Pt-Cl & Pt-Br & Pt-I \\
\hline$d_{1}(\AA)$ & 2.3274 & 2.4843 & 2.7918 \\
$d_{2}(\AA)$ & 3.1014 & 3.0013 & 3.0368 \\
$d_{2} / d_{1}$ & 1.33 & 1.21 & 1.09 \\
$\rho$ & 0.91 & 0.64 & 0.36 \\
$E_{\mathrm{CT}}(\mathrm{eV})$ & 2.72 & 1.95 & 1.37 \\
$E_{1 \mathrm{~m}}(\mathrm{eV})$ & 1.22 & 0.76 & 0.6 \\
$\omega_{\mathrm{R}}\left(\mathrm{cm}^{-1}\right)$ & 308 & 166 & 113 \\
$T_{\text {vib }}(\mathrm{fs})$ & 108 & 201 & 295 \\
\hline \hline
\end{tabular}


a pair of $\mathrm{Pt}^{3+}$ ions, i.e., a CT exciton. The peak energy of this absorption band $\left(E_{\mathrm{CT}}\right.$ in Table $\left.\mathrm{I}\right)$ becomes higher with decreasing the halogen ion radius because the displacement of halogen ions is responsible for the energy stabilization of electrons on $\mathrm{Pt}^{3-\rho}$ sites.

The photogenerated exciton is initially in the Bloch state, and its wave function extends over the $1 \mathrm{D}$ Pt- $X$ chain. However, the exciton immediately relaxes to the self-trapped state and causes the local lattice deformation because of the absence of energy barrier between the free and self-trapped states in 1D systems. ${ }^{8}$ As the positions of Pt ions are rather tightly fixed by the surrounding ligand molecules (ethylenediamine), the deformation of lattice in Pt- $X$ is chiefly the movement of halogen ions $X^{-}$. The $X^{-}$between the two $\mathrm{Pt}^{3+}$ ions is expected to be displaced to the position close to the middle point of the $\mathrm{Pt}^{3+}$ ions. When the crystals of $\mathrm{Pt}-X$ are excited at the CT absorption band or higher energy, intense Stokes-shifted broad luminescence bands due to the self-trapped exciton (STE) appear in the near-infrared wavelength region. ${ }^{9}$ This luminescence is known to be strongly polarized parallel to the chain axis of the crystal. In Table I, the luminescence peak energies are summarized $\left(E_{1 \mathrm{~m}}\right)$. The peak energy becomes lower with larger halogen ion, i.e., with lower CT gap energy. In addition, the frequency of the Raman-active symmetric stretching mode is shown in the table $\left(\omega_{\mathrm{R}}\right)$. It naturally becomes lower with increasing the halogen ion mass.

In this paper, we report the results of femtosecond timeresolved measurement of the STE luminescence in Pt- $X$. In $\mathrm{Pt}-\mathrm{Cl}$, new short-lived hot luminescence originating from the excited vibrational states of the STE is found in the lowenergy side of the STE luminescence band. The overall behavior of the STE luminescence within $2 \mathrm{ps}$ in $\mathrm{Pt}-\mathrm{Cl}$ is well understood by the vibrational relaxation of the STE. It is reproduced by a model calculation based on wave packet propagation on an interaction mode composed of frequencydispersed bulk phonons. This model is also applied to the previous results in $\mathrm{Pt}-\mathrm{Br},{ }^{10}$ and for both the systems, the frequency spectra of phonons which compose the interaction mode have been estimated. Meanwhile, the annihilation dynamics of the STE is investigated by observing the decay of the luminescence. In Pt-I, the STE decays much faster than in $\mathrm{Pt}-\mathrm{Cl}$ and $\mathrm{Pt}-\mathrm{Br}$.

\section{EXPERIMENT}

The time evolution of luminescence has been investigated by the frequency up-conversion technique. The light source is a mode-locked Ti:sapphire laser (central photon energy 1.6 $\mathrm{eV}$, average power $900 \mathrm{~mW}$, repetition rate $90 \mathrm{MHz}$, pulse width $60 \mathrm{fs}$ ). A schematic diagram of the measurement system is shown in Fig. 1. The output beam of the ultrashort pulse laser is divided into two beams. One of them is used for the excitation of a sample. It is either the fundamental frequency or the second harmonic (SH) light of the Ti:sapphire laser. The polarization direction of the excitation light is variably rotated by a half-wave plate (HWP1). The average excitation power is reduced to about $1 \mathrm{~mW}$ (about $3 \mathrm{~W} / \mathrm{cm}^{2}$ ) by a neutral density filter so as to prevent the

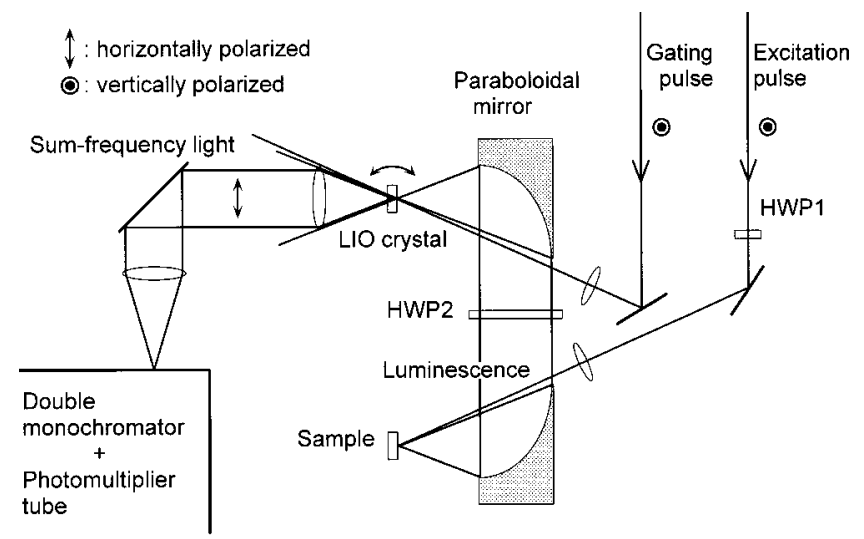

FIG. 1. Schematic diagram of the measurement system. HWP1 and 2 are half-wave plates.

damage of the sample. The luminescence of the sample is collected and focused onto a nonlinear optical crystal $\left(\mathrm{LiIO}_{3}\right.$, thickness 0.5 or $1 \mathrm{~mm}$ ) by off-axis paraboloidal mirrors. Another beam of the Ti:sapphire laser ("gating pulse" in the figure) is suitably delayed and focused onto the same nonlinear crystal. The time-resolved luminescence is detected as sum frequency photons generated from the luminescence and the gating laser pulse. The sum frequency light is collected and focused into a double-grating monochromator, and is detected by a photomultiplier tube with a photon counter. The time evolution is measured by varying the time delay of the gating pulse with respect to the sample excitation pulse.

For the excitation of $\mathrm{Pt}-\mathrm{Cl}$ samples, the $\mathrm{SH}$ light of the Ti:sapphire laser (photon energy $3.2 \mathrm{eV}$ ) is used. The time resolution of the measurement system is about $160 \mathrm{fs}$ in this case. For the excitation of $\mathrm{Pt}-\mathrm{Br}$ samples, the fundamental frequency light $(1.6 \mathrm{eV})$ is used, and the time resolution is about 90 fs. In experiments with Pt-I samples, the output beam of a Ti:sapphire regenerative amplifier (central photon energy $1.6 \mathrm{eV}$, repetition rate $100 \mathrm{kHz}$, pulse width $100 \mathrm{fs}$ ) is used as a light source. In this case, the time resolution is rather worse and about $170 \mathrm{fs}$ due to the larger laser pulse width.

As will be described in the next section, the direction and degree of luminescence polarization are examined for Pt-I samples. For this purpose, another half-wave plate (HWP2) is placed between the paraboloidal mirrors. As only the polarization component of luminescence satisfying the phasematching condition for sum frequency generation is frequency up-converted, the nonlinear crystal acts as a polarization filter. Thus, by variably rotating the polarization direction of luminescence by HWP2 and measuring the intensity of sum frequency signals, we can analyze the direction and degree of polarization of the luminescence.

All the experiments have been done with as-grown single crystals of Pt- $X$. In this study, the Pt- $X$ samples are always kept at room temperature $(296 \mathrm{~K})$ in experiments.

\section{RESULTS AND DISCUSSION}

\section{A. Model of hot luminescence}

First of all, we describe the model to calculate the time dependence of luminescence. By comparing the calculated 
results and the experimental ones, we can obtain the frequency spectra of phonons which compose the interaction mode of the self-trapping process.

We consider a two-level system of an electron coupled with bulk phonons through a linear interaction. In the strong coupling limit, the spectrum of transient hot luminescence in the system is given by Kayanuma ${ }^{11}$ as

$$
F_{e}\left(\Omega_{2}, \Omega_{1} ; t\right)=\frac{\sqrt{2 \pi}}{\widetilde{D}(t)} \exp \left[-\frac{\left\{\Omega_{2}-\tilde{\boldsymbol{\epsilon}}(t)\right\}^{2}}{2 \widetilde{D}(t)^{2}}\right] .
$$

Here, $\Omega_{1}$ and $\Omega_{2}$ are incident and scattered photon energies, respectively. $\tilde{\boldsymbol{\epsilon}}(t)$ is the time-dependent Franck-Condon energy and given as,

$$
\tilde{\epsilon}(t)=\epsilon-2 E_{\mathrm{LR}}+2 \int_{-\infty}^{\infty}\left\{\omega^{-1}+\frac{\Omega_{1}-\epsilon}{2 D^{2}}\right\} J(\omega) \cos \omega t d \omega .
$$

$\epsilon$ is the electronic excitation energy and $E_{\mathrm{LR}}$ is the lattice relaxation energy. $D$ represents the spectral width of the linear absorption (or luminescence) band. $J(\omega)$ is the spectral density function, which defines the frequency spectrum of phonons coupled to the electronic state. $\widetilde{D}(t)$ expresses the time dependence of spectral width of the hot luminescence and is given as

$$
\widetilde{D}(t)^{2}=D^{2}-D^{-2}\left\{\int_{-\infty}^{\infty} J(\omega) \cos \omega t d \omega\right\}^{2} .
$$

For strong electron-phonon coupling, it is necessary that the electron interacts with phonons of a wide range of the Brillouin zone. For this sake, the spatial size of the electronic wave function need to be of the order of a unit cell. Under this situation, the interaction mode consists of the modes continuously distributed within a finite frequency width reflecting the frequency dispersion of phonons. Then, the spectrum $J(\omega)$ is composed of continuous bands of finite widths.

For the fitting to the experimental results, we simplify the above three equations in the following way. First, Eq. (1) is modified as

$$
F_{e}\left(\Omega_{2}, t\right)=\frac{\sqrt{2 \pi}}{\widetilde{D}(t)} \exp \left[-\frac{\left\{\Omega_{2}-\tilde{\epsilon}(t)\right\}^{2}}{2 \widetilde{D}(t)^{2}}-\frac{t}{\tau}\right] .
$$

The last factor $\exp (-t / \tau)$ represents the decay of luminescence, i.e., the annihilation of STE's. $\tau$ is the lifetime of STE. Second, the time-dependent Franck-Condon energy $\tilde{\boldsymbol{\epsilon}}(t)$ is modified as

$$
\tilde{\epsilon}(t)=\epsilon-2 E_{\mathrm{LR}}+2 E_{\mathrm{LR}} \int_{-\infty}^{\infty} J^{\prime}(\omega) \cos \omega t d \omega .
$$

The function $J^{\prime}(\omega)$ is dimensionless normalized spectral density and has to satisfy the following condition;

$$
\int_{-\infty}^{\infty} J^{\prime}(\omega) d \omega=1
$$

The term $\left(\Omega_{1}-\epsilon\right) / 2 D^{2}$ in Eq. (2) is neglected because the excitation photon energy is always in the CT absorption band in this study $\left(\Omega_{1} \approx \epsilon\right)$. Thus, $J^{\prime}(\omega)$ has the relationship $J^{\prime}(\omega) \propto J(\omega) / \omega$. The time dependence of spectral width $\widetilde{D}(t)$ is expressed as

$$
\widetilde{D}(t)=D_{0}+D_{1} \sqrt{1-\left\{\int_{-\infty}^{\infty} J^{\prime}(\omega) \cos \omega t d \omega\right\}^{2}} .
$$

A constant term $D_{0}$ is added. This represents broadening due to the finite spectral width of the excitation laser pulse.

When the spectrum $J^{\prime}(\omega)$ is composed of continuous bands of finite widths, the relation

$$
\lim _{t \rightarrow \infty} \int_{-\infty}^{\infty} J^{\prime}(\omega) \cos \omega t d \omega=0
$$

is satisfied because of dephasing among different frequency components at large $t$. Then, the relations

$$
\begin{gathered}
\lim _{t \rightarrow 0} \tilde{\epsilon}(t)=\epsilon, \quad \lim _{t \rightarrow 0} \frac{d}{d t} \tilde{\epsilon}(t)=0, \quad \lim _{t \rightarrow \infty} \tilde{\epsilon}(t)=\epsilon-2 E_{\mathrm{LR}}, \\
\lim _{t \rightarrow 0} \widetilde{D}(t)=D_{0}, \quad \lim _{t \rightarrow \infty} \widetilde{D}(t)=D_{0}+D_{1}
\end{gathered}
$$

are satisfied. All the calculations based on the model have been performed with the convolution of Eq. (4) with the response function of the measurement system.

In the previous reports, ${ }^{10,12}$ different models from the above one are applied to Pt-Cl and Pt-Br. The model described here can, however, be applied to both the systems. It manifests naturally both the energy and phase relaxation of a vibrational state without defining a phenomenological energy-dissipation rate.

\section{B. Pt-Br}

Here we apply the model to the previous results in Pt-Br. ${ }^{10}$ In the experiment, the samples of $\mathrm{Pt}-\mathrm{Br}$ at room temperature are excited at $1.6 \mathrm{eV}$. This photon energy corresponds to the low-energy tail of the CT absorption band. The excitation light is polarized parallel to the chain axis of the crystal. It produces the free excitons with small kinetic energy. The luminescence is observed over the photon energy range from 0.7 to $1.4 \mathrm{eV}$ which includes the peak of the STE luminescence band of about $0.8 \mathrm{eV}$. The polarization component of luminescence parallel to the chain axis is observed.

The time evolution of luminescence is shown in Fig. 2. It shows strong dependence on detection photon energy. The calculated results are shown as dotted curves in the figure. The lattice relaxation is assumed to start at $t=0$. In the calculation, the parameters shown in Table II are used. These values are determined as follows. The electronic excitation energy $\epsilon$ is given by the position of the low-energy edge of the $\mathrm{CT}$ absorption band. The lattice relaxation energy $E_{\mathrm{LR}}$ is determined from the luminescence peak energy $0.8 \mathrm{eV}$ $\left(=\epsilon-2 E_{\mathrm{LR}}\right)$. The initial spectral width of hot luminescence $D_{0}$ is given by the transform-limited band width of the $50 \mathrm{fs}$ excitation light pulse. The final spectral width $D_{0}+D_{1}$ is 


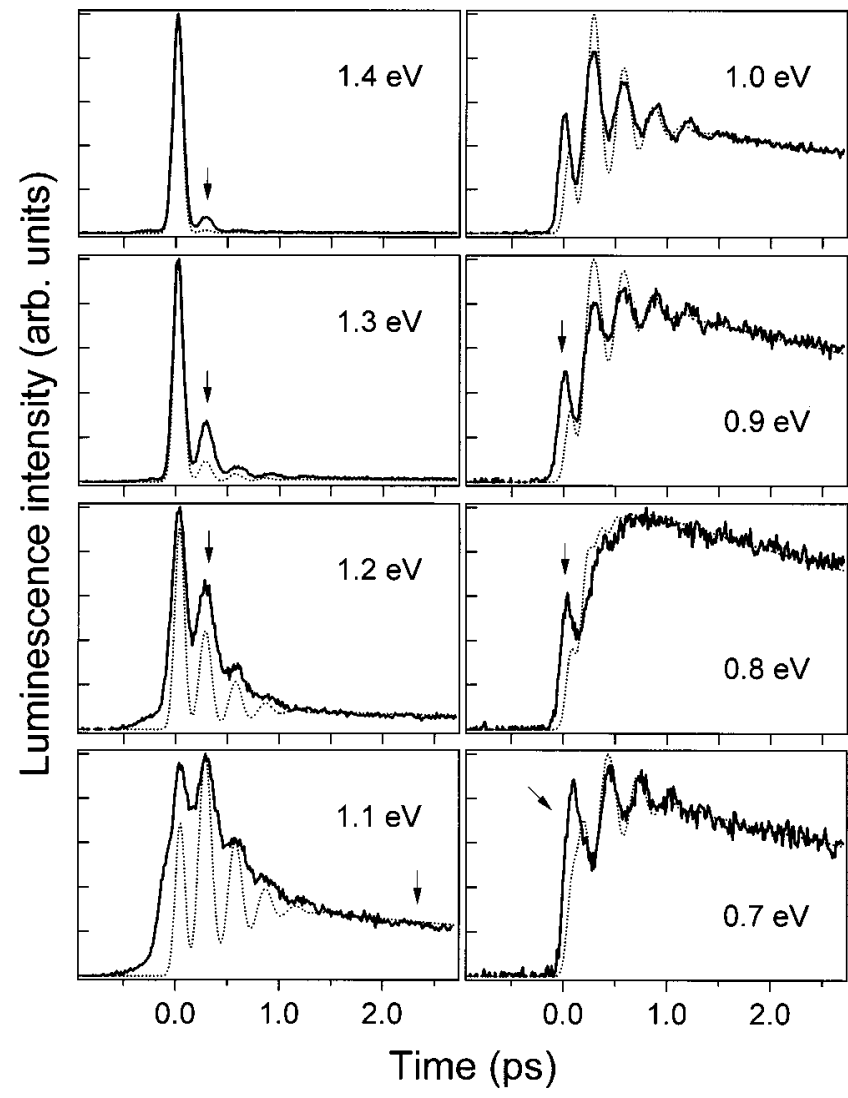

FIG. 2. Time evolution of luminescence in $\mathrm{Pt}-\mathrm{Br}$ at indicated emission photon energies. The experimental and calculated results are shown as solid and dotted lines, respectively. Refer to the text for the meanings of arrows.

determined from the fitting to the experimental data. The obtained value of $0.155 \mathrm{eV}$ corresponds to the FWHM of about $0.37 \mathrm{eV}$. As the STE luminescence band in $\mathrm{Pt}-\mathrm{Br}$ has the spectral width of about $0.26 \mathrm{eV}(\mathrm{FWHM})$ at $2 \mathrm{~K},{ }^{13}$ the value of $0.37 \mathrm{eV}$ is quite reasonable when we take account of thermal broadening at room temperature. The lifetime of

TABLE II. Values of fitting parameters used for $\mathrm{Pt}-\mathrm{Br}$ and $\mathrm{Pt}-\mathrm{Cl}$ in the calculation. $\epsilon$ is the electronic excitation energy. $E_{\mathrm{LR}}$ is the lattice relaxation energy. $D_{0}$ and $D_{0}+D_{1}$ are the initial and the final spectral widths of hot luminescence, respectively. $\tau$ is the lifetime of STE. $T$ is the oscillation period at the central phonon frequency. $W_{1}$ and $W_{2}$ are the frequency widths of the first and second Gaussian components. $a$ is the weight of the second component.

\begin{tabular}{lcc}
\hline \hline \multicolumn{1}{c}{ Parameter } & Pt-Br & Pt-Cl \\
\hline$\epsilon(\mathrm{eV})$ & 1.4 & 2.6 \\
$E_{\mathrm{LR}}(\mathrm{eV})$ & 0.3 & 0.66 \\
$D_{0}(\mathrm{meV})$ & 15 & 15 \\
$D_{0}+D_{1}(\mathrm{eV})$ & 0.155 & 0.235 \\
$\tau(\mathrm{ps})$ & 6 & 25 \\
$T=2 \pi / \omega_{c}(\mathrm{fs})$ & 290 & 140 \\
$W_{1}$ & $2 \omega_{c} / 3$ & $2 \omega_{c} / 9$ \\
$W_{2}$ & $2 \omega_{c} / 14$ & $2 \omega_{c} / 42$ \\
$a$ & 0.3 & 0.5 \\
\hline \hline
\end{tabular}

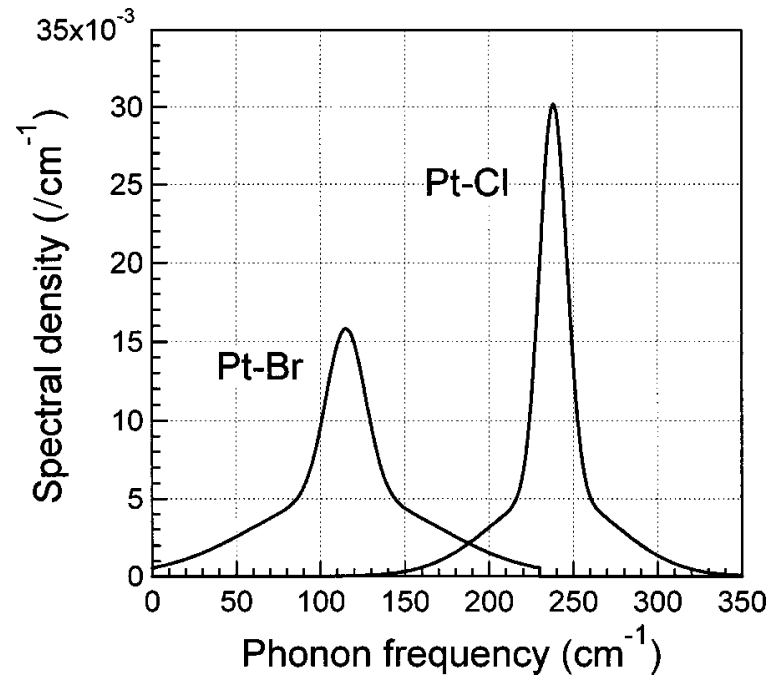

FIG. 3. Spectral density function, $J^{\prime}(\omega)$, assumed in the calculation of the time-resolved luminescence in $\mathrm{Pt}-\mathrm{Br}$ and $\mathrm{Pt}-\mathrm{Cl}$.

STE $\tau$ is determined from the luminescence decay (5.5 $\pm 1 \mathrm{ps}) .{ }^{10}$ To reduce the degree of freedom in $J^{\prime}(\omega)$, we have to assume a specific function shape appropriately. We have tried several types of $J^{\prime}(\omega)$, and have found the following form which consists of two Gaussian components with the same center of gravity is appropriate.

$$
J^{\prime}(\omega)=\left\{\begin{array}{r}
\frac{1-a}{\sqrt{\pi} W_{1}} \exp \left[-\frac{\left(\omega-\omega_{c}\right)^{2}}{W_{1}^{2}}\right] \\
+\frac{a}{\sqrt{\pi} W_{2}} \exp \left[-\frac{\left(\omega-\omega_{c}\right)^{2}}{W_{2}^{2}}\right], \\
0 \leqslant \omega \leqslant 2 \omega_{c}, \\
0, \quad \text { otherwise. }
\end{array}\right.
$$

Here $\omega_{c}$ is the center of the phonon frequency. The frequency width $W_{1}$ is assumed to be larger than the other one $W_{2}$. Parameter $a$ represents the weight of the contribution of the second component. These four parameters determine the shape of $J^{\prime}(\omega)$. Their values are determined from the fitting to the experimental data as shown in Table II. The obtained $J^{\prime}(\omega)$ is plotted in Fig. 3. The time origin of the experimental data in Fig. 2 is determined by the fitting with the calculated results.

As seen in Fig. 2, the model reproduces the experimental results very well. We can see a distinct temporal oscillation with a period of about 290 fs. This period determines the value of $T\left(=2 \pi / \omega_{c}\right)$. At 1.4 and $1.3 \mathrm{eV}$, the intensity of the second oscillation peak (indicated by arrows) is very weak, compared with that of the first one in both the experimental and calculated results. This shows the wave packet returning from the first turning point can not get back to the same position for these high transition energies. This can be seen in Fig. 4, where the energy position of the wave packet [the time-dependent Franck-Condon energy $\tilde{\boldsymbol{\epsilon}}(t)]$ is plotted as a function of time $t$. The fast damping of the oscillation in $\tilde{\boldsymbol{\epsilon}}(t)$ arises from the contribution of the first Gaussian component 


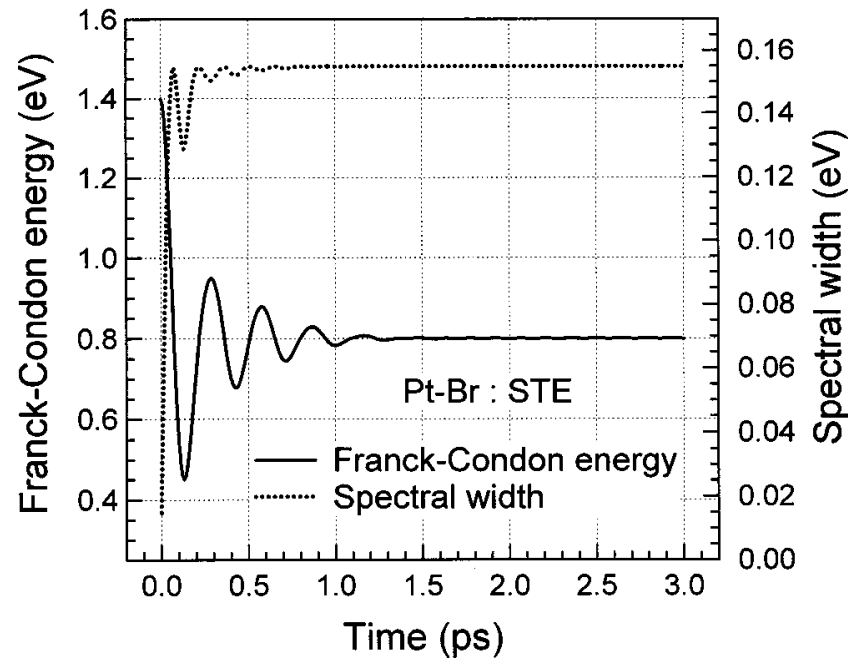

FIG. 4. Time-dependent Franck-Condon energy $\tilde{\boldsymbol{\epsilon}}(t)$ (solid curve) and time dependence of spectral width $\widetilde{D}(t)$ (dashed curve) calculated for Pt-Br.

of the broader spectral width $W_{1}\left(=2 \omega_{c} / 3\right)$ in $J^{\prime}(\omega)$. If $J^{\prime}(\omega)$ did not include this component, the second peak in the calculation would be much stronger than the experimental result.

The relative intensity of the second peak increases with decrease of the emission photon energy. At $1.1 \mathrm{eV}$, it becomes stronger than the first peak. This is reproduced by the calculation. The relative intensity of the second peak to the other ones becomes maximum at $1.0 \mathrm{eV}$. This means the center of the wave packet has the second turning point around the position for this transition energy. This is shown in Fig. 4. In the same way, the third peak appears and increases its intensity with decrease of the photon energy. As indicated by an arrow at $1.1 \mathrm{eV}$, the oscillation is followed by the slow decay with a lifetime of about 5.5 ps. $^{10}$

At 0.9 and $1.0 \mathrm{eV}$, the oscillation lasts at least for five periods. To reproduce this fact in the calculation, the second Gaussian component of the narrower frequency width $W_{2}$ is included in $J^{\prime}(\omega)$. The width is determined uniquely to be $2 \omega_{c} / 14$. As the energies are close to the transition energy from the bottom of the STE potential well, the luminescence intensity does not show fast decay.

At $0.8 \mathrm{eV}$, the oscillation disappears. It is reproduced well by the calculation. At this energy, only one oscillation peak indicated by an arrow is observed. This photon energy is equal to the peak of the stationary luminescence. The disappearance of the oscillation at the peak can be understood as follows. As the period of the wave packet oscillation is about $290 \mathrm{fs}$, the wave packet goes through the potential minimum every $1 \underset{\sim}{4} 5$ fs. Actually, the time-dependent Franck-Condon energy $\tilde{\epsilon}(t)$ in Fig. 4 passes through the energy of $0.8 \mathrm{eV}$ every $145 \mathrm{fs}$. Thus, if the time resolution of the measurement was sufficiently good, the oscillation of the period of $145 \mathrm{fs}$ would be observed in the luminescence at $0.8 \mathrm{eV}$. This oscillation is, however, invisible in the experiment because half the period (72.5 fs) is shorter than the time resolution (90 fs). At $0.8 \mathrm{eV}$, only one oscillation peak indicated by an arrow is observed at the very early time. It is due to the first transit of

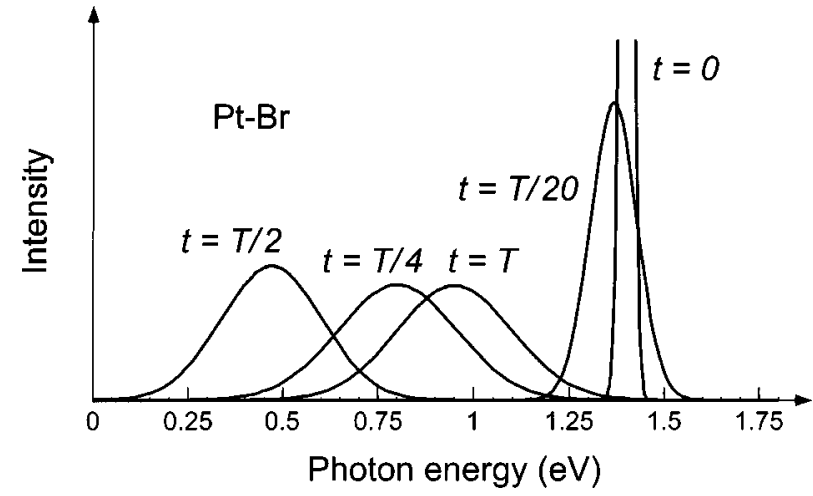

FIG. 5. Time-resolved hot luminescence spectra calculated with parameter values in Table II for Pt-Br. The spectra at $t=0, T / 20$, $T / 4, T / 2$, and $T$ are shown. $T$ is the period of the wave packet oscillation (290 fs). The abscissa represents the luminescence photon energy.

the wave packet at the potential minimum, and reproduced in the calculation as a small hump.

At $0.7 \mathrm{eV}$, the oscillation appears again both in the experimental and calculation data. At this energy, the oscillation lasts at least for three periods. It can be seen in the experimental data that the intensity of the first oscillation peak (indicated by an arrow) is as strong as that of the slow decay component. It is reproduced well by the calculation. At 0.9 $\mathrm{eV}$, the intensity of the first peak (indicated by an arrow) was about half of the slow decay component intensity. This is also reproduced by the calculation. By assuming the appropriate spectral density function $J^{\prime}(\omega)$, the model has reproduced the above many important features of the experimental results.

Figure 5 shows the time-resolved hot luminescence spectra calculated from the model with the parameters shown in Table II. At $t=0$, the spectral width is as narrow as the band width of the excitation light pulse. It, however, broadens out very rapidly and becomes maximum at $t=T / 4(=72.5 \mathrm{fs})$ when the wave packet passes through the potential minimum. Then the width narrows slightly, and the wave packet reaches the first turning point at $t=T / 2$ (=145 fs). After one oscillation period [at $t=T(=290 \mathrm{fs})]$, the spectral width becomes much broader than the initial one. The time dependence of the spectral width $\widetilde{D}(t)$ is plotted as a dashed curve in Fig. 4. The spatial size of the electronic wave function shrinks simultaneously with the first cycle of the wave packet oscillation along the interaction mode. Thus, this mode is responsible for triggering the localization of the electronic wave function and for energetic stabilization of the localized state.

Tanaka and Kayanuma ${ }^{14}$ theoretically investigated the effect of exciton transfer on the self-trapping dynamics in a 1D system, and calculated the time evolution of luminescence. In the large exciton transfer case, the peak position of the hot luminescence spectrum stands still around the excitation photon energy for a moment, and then the damped oscillation starts. This reflects that the exciton remains free for a while after the photoexcitation owing to the effect of the transfer. 
Thus, the transfer may influence on the starting time of the damped oscillation which is not determined experimentally in this study.

Here we discuss the phonon modes which contribute to the interaction mode. In Pt-Br, the frequency of the Ramanactive symmetric stretching mode is $166 \mathrm{~cm}^{-1}$. The frequency dispersion of this mode is known to be very small. ${ }^{15}$ Thus this frequency is too high to explain the observed value $115 \mathrm{~cm}^{-1}$ (the period $T=290 \mathrm{fs}$ ). In FIR reflectivity ${ }^{16,17}$ and transmittance ${ }^{18}$ spectra of Pt-Br, several vibration modes appear around $115 \mathrm{~cm}^{-1}$. Although their origin is not clear, it is possible that they contribute to the interaction mode. There is another possibility which seems to be more realistic. The dispersion relation of vibration in Pt- $X$ chain is calculated by Bulou et al. ${ }^{15}$ It shows that two dispersive modes lie at moderately lower frequency than that of the symmetric stretching mode. They may contribute to the interaction mode. As the exciton wave function becomes localized in the spatial size of the order of a unit cell, it can be coupled to the modes throughout the Brillouin zone. As shown in Fig. 3, the interaction mode has the broad spectral width. It might be due to the dispersion of those modes.

Dexheimer et al. ${ }^{19}$ observed a temporal oscillation in the time-resolved transmittance of $\left[\mathrm{Pt}(\mathrm{en})_{2}\right]\left[\mathrm{Pt}(\mathrm{en})_{2} \mathrm{Br}_{2}\right]\left(\mathrm{PF}_{6}\right)_{4}$ (abbreviated as $\mathrm{Pt}-\mathrm{Br} / \mathrm{PF}$ below). This system consists of the same chain structure as $\mathrm{Pt}-\mathrm{Br}$ of alternating $\mathrm{Pt}$ and $\mathrm{Br}$ ions. The Fourier power spectra of the data shows two peaks at 110 and $175 \mathrm{~cm}^{-1}$. The peak at $175 \mathrm{~cm}^{-1}$ corresponds to the symmetric stretching mode in $\mathrm{Pt}-\mathrm{Br} / \mathrm{PF}$. It should be noted that the frequency is very close to that in $\mathrm{Pt}-\mathrm{Br}$ (Table I). The peak at $110 \mathrm{~cm}^{-1}$ corresponds to the self-trapping mode. This frequency is very close to that in $\mathrm{Pt}-\mathrm{Br}$ $\left(115 \mathrm{~cm}^{-1}\right)$. The oscillation of the mode decays very fast and disappears after $1 \mathrm{ps}$. This fact is in good agreement with our previous result in Pt-Br. ${ }^{10}$

\section{Pt-Cl}

In the previous report on $\mathrm{Pt}-\mathrm{Cl},{ }^{12}$ the detection energy range was limited above $0.95 \mathrm{eV}$. By observing the luminescence below $0.95 \mathrm{eV}$, we have found a new fast decay component. Here, we report the overall behavior of luminescence which include the new fast component in the low-energy side. In the experiment, the sample at room temperature is excited at $3.2 \mathrm{eV}$ by the light polarized parallel to the chain axis. The excitation energy is in the high-energy side of the $\mathrm{CT}$ absorption band. It produces the excitons with large excess kinetic energy. The polarization component of luminescence parallel to the chain is observed.

Figure 6 shows the time evolution of luminescence at the emission photon energies from 0.85 to $2.1 \mathrm{eV}$. Around the peak of the band $(1.2 \mathrm{eV})$, the luminescence decays slowly. The lifetime of the slow decay component is $30 \pm 10$ ps. ${ }^{12} \mathrm{In}$ addition, a fast decay component appears at the high-energy tail of the band (above $1.8 \mathrm{eV}$ ). It decays within $1 \mathrm{ps}$ and becomes faster in higher energies. The intensity of the fast decay component relative to the slow one increases with increasing emission photon energy. Around the peak energy $(1.2 \mathrm{eV})$, the luminescence rises slowly. As can be seen in the

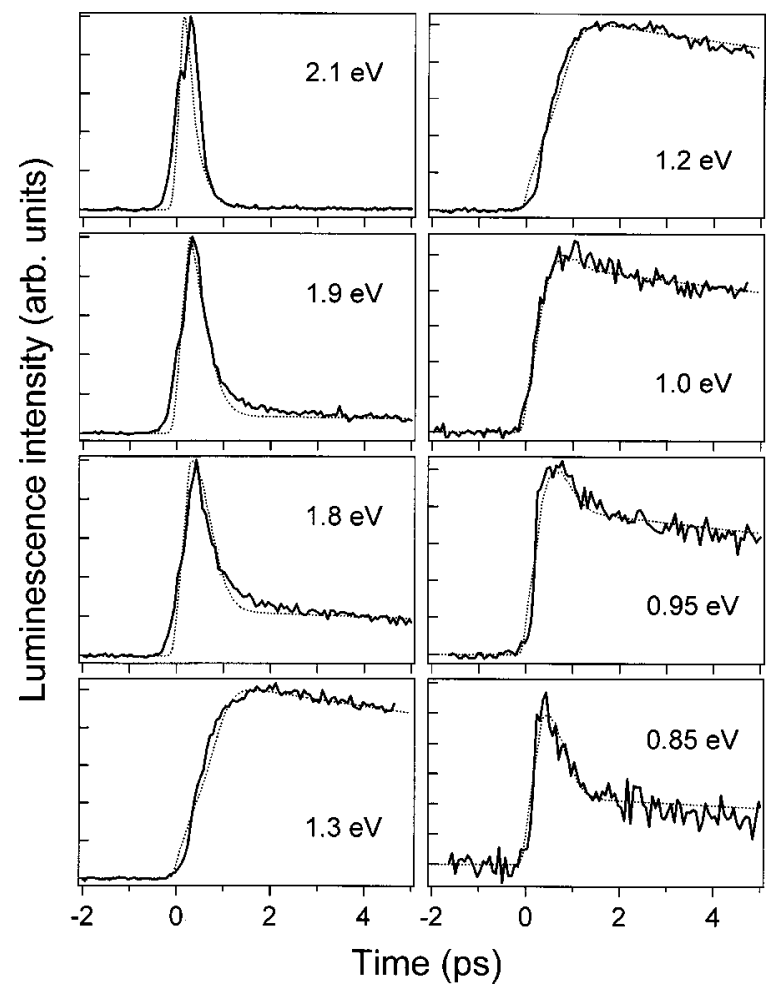

FIG. 6. Time evolution of luminescence in $\mathrm{Pt}-\mathrm{Cl}$ at indicated emission photon energies. The experimental and calculated results are shown as solid and dotted lines, respectively. The luminescence in the energy range between 1.4 and $1.8 \mathrm{eV}$ could not be observed because of the strong stray light of SH generated at the nonlinear crystal.

figure, this rise becomes faster in the low-energy side of the luminescence band. The rise below $0.91 \mathrm{eV}$ is very fast and limited by the experimental time resolution (160 fs). In the data below $0.95 \mathrm{eV}$, we can find the appearance of a fast decay component of the lifetime of about $1 \mathrm{ps}$. As seen in the figure, the intensity of this component relative to the slow decay component becomes stronger at lower photon energy. The lifetime is very close to that of the fast component in the high-energy side, and becomes shorter with decrease of the detection energy.

We apply the model to the present result in Pt-Cl. The calculated results are shown in Fig. 6 as dotted curves. The values of fitting parameters used in the calculation are shown in Table II. The electronic excitation energy $\epsilon$ is determined to be $2.6 \mathrm{eV}$. This energy is in the low-energy side of the CT absorption band. The lattice relaxation energy $E_{\mathrm{LR}}$ is determined to be $0.66 \mathrm{eV}$. The $\epsilon$ and $E_{\mathrm{LR}}$ give the stationary luminescence peak at $1.28 \mathrm{eV}\left(=\epsilon-2 E_{\mathrm{LR}}\right)$, which is very close to the reported value. ${ }^{2}$ The initial spectral width of hot luminescence $D_{0}$ is given roughly by the band width of the excitation light pulse. The final spectral width $D_{0}+D_{1}$ is determined to be $0.235 \mathrm{eV}$. This width is equal to the FWHM of about $0.55 \mathrm{eV}$, and is close to the real width of the stationary luminescence in $\mathrm{Pt}-\mathrm{Cl}$ at room temperature. The lifetime of STE $\tau$ is experimentally determined. ${ }^{12}$ The oscillation period $T$ at the central phonon frequency $\omega_{c}$ is assumed to be $140 \mathrm{fs}$. This period is a little longer than the period of 
the symmetric stretching mode in $\mathrm{Pt}-\mathrm{Cl}$ (Table I). It is assumed from the analogy of $\mathrm{Pt}-\mathrm{Br}$ in which the oscillation period $T$ is a little longer than that of the symmetric stretching mode. The other three parameters $W_{1}, W_{2}$, and $a$ have to be determined from the fitting of the calculation data to the experimental results. They determine the shape of the function $J^{\prime}(\omega)$. Here, to reduce the degree of freedom, the ratio $W_{1} / W_{2}=14 / 3$ is assumed from the analogy of the result in $\mathrm{Pt}-\mathrm{Br}$. This assumption enables us to obtain unique values of $a$ and $W_{1}\left(\right.$ and $\left.W_{2}\right)$. The obtained $J^{\prime}(\omega)$ spectrum is shown in Fig. 3. The model calculation is performed with the convolution with the system response function.

As shown in Fig. 6, the calculated results are in good agreement with the experimental ones. They have reproduced the following important features of the experimental data: (1) the fast rise and decay in the high-energy tail of the luminescence band (above $1.8 \mathrm{eV}$ ), (2) the slow rise around the luminescence peak $(1.2 \mathrm{eV}),(3)$ the faster rise at lower energy in the low energy side of the luminescence band (below $1.2 \mathrm{eV})$, (4) the appearance of the fast decay component in the low-energy tail of the luminescence band (below 0.95 $\mathrm{eV}$ ), and (5) the intensity ratio between the fast and slow decay components. The excellent agreement with the experimental data, which includes the presence of the fast decay component in the low-energy side of the band, assures us that the observed time dependence within 2 ps reflects the lattice relaxation of excitons. The system during the process is expressed as the oscillating wave packet on the interaction mode. However, it is not observed explicitly. Probably it is because the oscillation period is shorter than the experimental time resolution (160 fs).

\section{Pt-I}

Here, we describe the experimental results in Pt-I. In the experiment, the samples at room temperature are excited at $1.6 \mathrm{eV}$. This energy is at the high-energy side of the CT absorption band.

Figure 7 shows the time evolution of luminescence in Pt-I at various photon energies from 0.7 to $1.4 \mathrm{eV}$. The experimental data are shown by dots in the figure. In the measurement of the data in Fig. 7, the excitation light is polarized along the chain axis of the crystal, and the polarization component of luminescence parallel to the chain is observed. The time dependence can be expressed by the double exponential decay $I(t)=\left(1-e^{-t / \tau_{r}}\right)\left(I_{f} e^{-t / \tau_{f}}+I_{s} e^{-t / \tau_{s}}\right)$. The fitting curves are shown in the figure by solid ones. The faster decay component has a lifetime $\tau_{f}$ of about $650 \mathrm{fs}$. This component is observed all over the detection energy range. The small deviations of the fitting curves from the experimental data before $t=0$ are probably due to an experimental artifact. They, however, does not affect the estimation of the lifetimes $\tau_{f}$ and $\tau_{s}$ which are sufficiently long. The dependence of $\tau_{f}$ on the emission photon energy is shown in Fig. 8.

The observed time evolution of luminescence in Pt-I is very different from those in $\mathrm{Pt}-\mathrm{Cl}$ and $\mathrm{Pt}-\mathrm{Br}$ where relatively slow decay is observed around the peak. In contrast, we can not observe such slow decay even at energies close to the peak $(0.6 \mathrm{eV})$. To investigate the origin of the fast decay

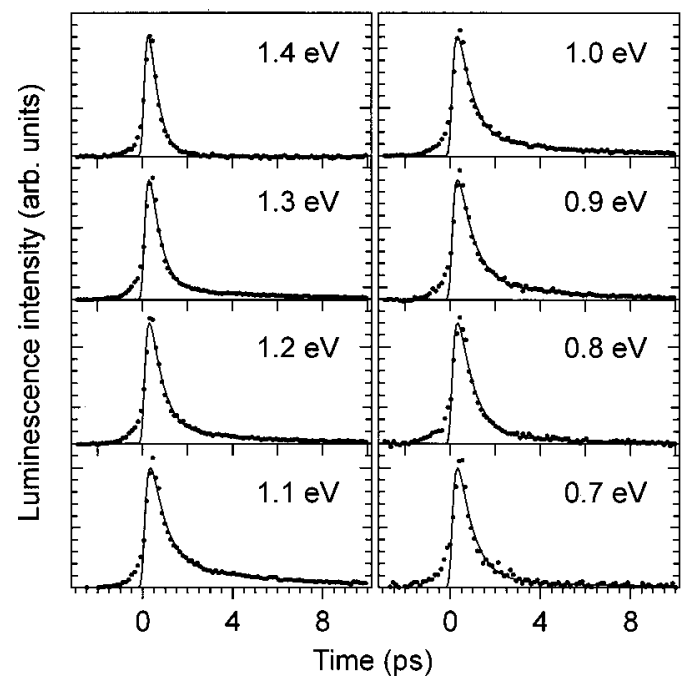

FIG. 7. Time evolution of luminescence in Pt-I. The emission photon energy is indicated for each data. The dots are experimental data. The solid curves are fitting ones that assume double exponential decay. They are obtained from the convolution with the system response function. The sample is excited by the light polarized along the chain axis $\left(\theta_{\mathrm{exc}}=0^{\circ}\right.$, refer to the inset in Fig. 9.). The polarization component of luminescence parallel to the chain is observed $\left(\theta_{\text {lum }}=0^{\circ}\right)$.

component $\left(\tau_{f}=650 \mathrm{fs}\right)$, the dependence of the luminescence on the excitation polarization direction is observed as shown in Fig. 9. In the measurement, the polarization component of luminescence parallel to the chain axis is observed $\left(\theta_{\text {lum }}=0^{\circ}\right)$. We will use below the angles $\theta_{\text {lum }}$ (luminescence polarization direction) and $\theta_{\text {exc }}$ (excitation polarization direction) which are defined by an inset in Fig. 9. As can be seen in the figure, the fast decay component does not appear at all under the excitation polarized perpendicular to the chain axis $\left(\theta_{\text {exc }}=90^{\circ}\right)$. As the excitation energy of $1.6 \mathrm{eV}$ is in the high-energy side of the CT absorption band which is polarized strongly along the chain axis, this data shows the initial state of luminescence of the fast decay component is created from CT excitons on the chain.

Next, the polarization of luminescence itself is examined. The time evolution of luminescence at $0.95 \mathrm{eV}$ of several

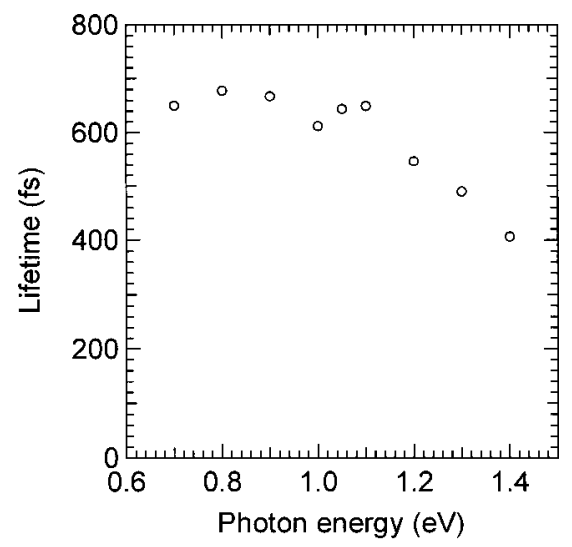

FIG. 8. Emission photon energy dependence of the lifetime $\tau_{f}$ of the fast decay component in Pt-I. 


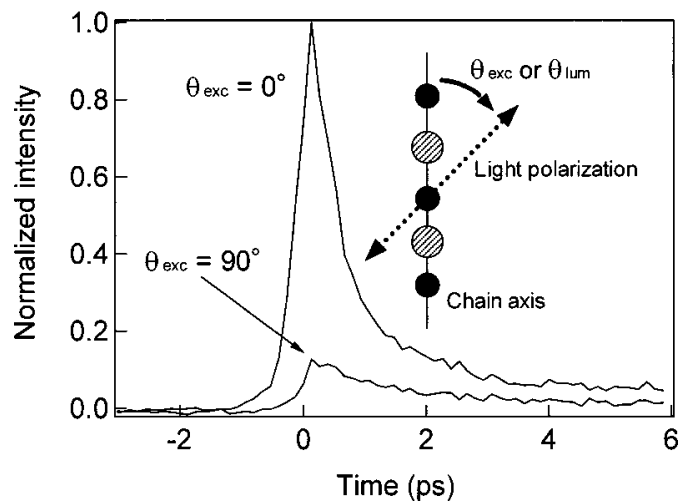

FIG. 9. Time evolution of luminescence at $0.95 \mathrm{eV}$ in Pt-I for two different excitation polarization directions $\left(\theta_{\mathrm{exc}}=0^{\circ}\right.$ and $\left.90^{\circ}\right)$. The polarization component of luminescence parallel to the chain is observed $\left(\theta_{\text {lum }}=0^{\circ}\right)$. The $\theta_{\text {exc }}$ and $\theta_{\text {lum }}$ are the angles of the excitation polarization direction and the luminescence polarization direction, respectively. They are defined in the inset where the Pt-I chain is illustrated.

different polarization directions $\theta_{\text {lum }}\left(=0,30,60\right.$, and $\left.90^{\circ}\right)$ is shown in Fig. 10. The sample is excited by the light polarized at four different angles relative to the chain $\left(\theta_{\mathrm{exc}}=\right.$ $-45,0,45$, and $90^{\circ}$ ). As clearly seen in the figure, the fast decay component is strongly polarized along the chain axis independently of the excitation polarization direction. This shows the transition dipole moment of the fast decay component is always parallel to the chain axis. As this polarized luminescence has a very broad band (Fig. 7), it should be attributed to the recombination of localized CT excitons on the chain, i.e., STE's.

As the stationary luminescence of STE in Pt-I has the peak at about $0.6 \mathrm{eV}$ (Table I), the observed emission energy range (from 0.7 to $1.4 \mathrm{eV}$ ) corresponds to the high-energy side of the STE luminescence band. The lifetime $\tau_{f}$ of the fast decay component shown in Fig. 8 becomes shorter with

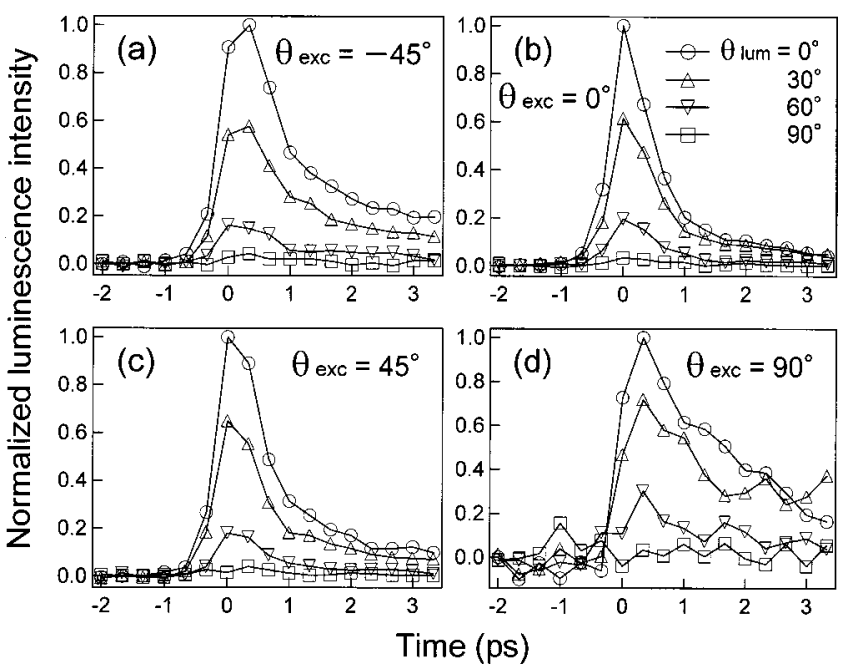

FIG. 10. Time evolution of luminescence in Pt-I at $0.95 \mathrm{eV}$ of several different polarization directions $\theta_{\text {lum }}\left(=0,30,60\right.$, and $\left.90^{\circ}\right)$. The excitation light is polarized at the angles $\theta_{\mathrm{exc}}=$ (a) $-45^{\circ}$, (b) $0^{\circ}$, (c) $45^{\circ}$, and (d) $90^{\circ}$.
TABLE III. Lifetimes of STE in Pt- $X$ at room temperature determined by the luminescence decay.

\begin{tabular}{lc}
\hline \hline Pt- $X$ & Lifetime $(\mathrm{ps})$ \\
\hline Pt-Cl & $30 \pm 10$ \\
Pt-Br & $5.5 \pm 1$ \\
Pt-I & $0.65 \pm 0.05$ \\
\hline
\end{tabular}

increasing the detection photon energy. It becomes about 400 fs at $1.4 \mathrm{eV}$. From the analogy of the results in $\mathrm{Pt}-\mathrm{Cl}$ and $\mathrm{Pt}-\mathrm{Br}$, we suppose that the shortening of the lifetime at higher photon energy reflects the vibrational relaxation of STE. The luminescence lifetime of $650 \pm 50$ fs near the peak energy $(0.6 \mathrm{eV})$ gives the lifetime of STE itself. Although we observed slow rise of luminescence around the peak due to the vibrational relaxation of STE in $\mathrm{Pt}-\mathrm{Cl}$ and $\mathrm{Pt}-\mathrm{Br}$, we do not observe it in Pt-I because the lifetime of STE itself is extremely short.

The STE lifetime in Pt-I at room temperature is recently reported to be much longer than a few picoseconds from the results of the time-resolved reflection spectroscopy. ${ }^{20} \mathrm{How}-$ ever, the lifetime determined here from the luminescence decay is much shorter (650 fs).

As can be seen in Fig. 7, an additional slow decay component with the lifetime $\tau_{s}$ of $5 \pm 1$ ps is observed around 1 $\mathrm{eV}$. This component is observed even under the excitation polarized perpendicular to the chain axis (Fig. 9, $\theta_{\mathrm{exc}}$ $=90^{\circ}$ ). The luminescence of the slow decay component is, however, strongly polarized along the chain axis (Fig. 10). At present, we tentatively attribute it to the luminescence of STE's perturbed by lattice imperfections or to the radiative recombination of polaronic defect states on the chain. It is because the relative intensity of this component to the fast decay component depends strongly on samples.

Although the phonon frequency is expected to be lower than those in $\mathrm{Pt}-\mathrm{Cl}$ and $\mathrm{Pt}-\mathrm{Br}$, we have not observed the wave packet oscillation in Pt-I. It might be due to the rather worse time resolution of the measurement (170 fs).

\section{E. Comparison of lifetimes of STE in Pt- $X$}

Lastly, we compare the lifetimes of STE in Pt- $X(X=\mathrm{Cl}$, $\mathrm{Br}$ or I). In Table III, the lifetimes at room temperature determined in this study are summarized.

The observed lifetimes should be ascribed to a nonradiative annihilation process, because the radiative lifetime of STE in Pt- $X$ has been estimated to be much longer (4-6 ns) from the oscillator strength of the CT transition. ${ }^{21}$ This process is a thermally activated one in $\mathrm{Pt}-\mathrm{Cl}$, as assured by the temperature dependence of the luminescence lifetime. ${ }^{22}$ As seen in the table, the STE lifetime depends strongly on the bridging-halogen ion. It becomes shorter with increasing the halogen ion radius. This fact is in good agreement with the stationary luminescence intensity of Pt- $X$ which becomes weaker with decrease of the CT gap energy. ${ }^{23}$

Iwano $^{24}$ theoretically investigated the relaxation process of STE in Pt- $X$ by calculating the adiabatic potential energy surfaces with the so-called 1D extended Peierls-Hubbard 


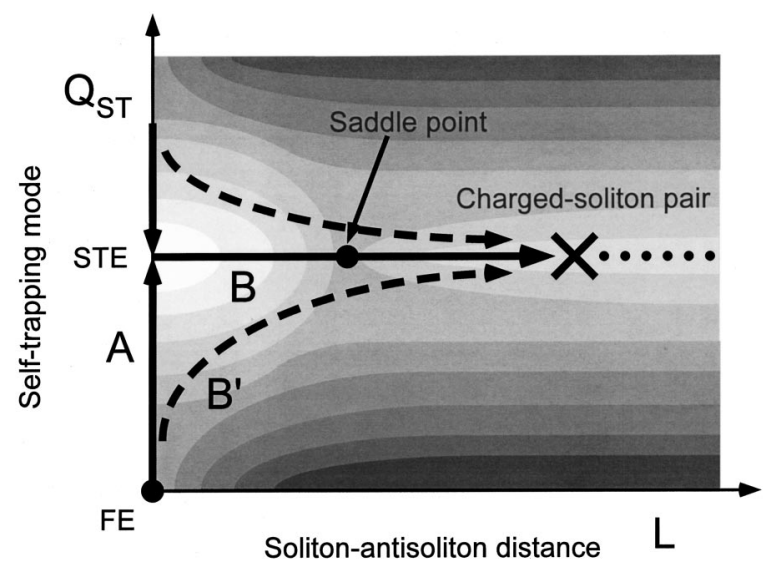

FIG. 11. Schematic diagram of the relaxation processes on the first excited state potential surface. $Q_{\mathrm{ST}}$ is the configuration coordinate of the interaction mode in the self-trapping process. $L$ expresses the distance between a soliton and an antisoliton. The potential energy of the darker area is higher. The vertical arrows mean the vibrational relaxation of STE (process A). The horizontal arrow is the relaxation from STE to soliton-pair state in which the system crosses over the potential barrier at the saddle point (process B). The dashed arrows express the relaxation to the soliton-pair state before the thermalization within the local potential minimum of STE (process B'). Most of the STE's nonradiatively return back to the ground state before complete dissociation to the soliton pairs as represented by a cross in the figure, and the rest become persistent soliton pairs (dotted line) (Refs. 23,24).

model. With moderate values of material parameters, he showed that the charged-soliton-pair state is on the same potential energy surface as the STE state. On the other hand, the ground state (the uniform CDW state) is connected to the spin-soliton-pair state. The potential energy surface of the first excited state is illustrated in Fig. 11. The vertical axis $Q_{\mathrm{ST}}$ is the configuration coordinate of the interaction mode in the self-trapping process. The horizontal axis $L$ expresses the distance between a soliton and an antisoliton. The relaxation along this axis means the dissociation of exciton to the charged-soliton pair, which is possible in the doubly degenerate $1 \mathrm{D}$ CDW state. The system can return nonradiatively to the ground state via the soliton-pair state which is separated from the STE state by a small energy barrier. This relaxation process has been proposed for the excited-state dynamics in relative systems of $\mathrm{Pt}-X{ }^{23}$ It is indicated by a solid arrow $\mathrm{B}$ in the figure. The stability of the STE in Pt- $X$ depends on the energy barrier height at the saddle point and is reflected in the luminescence lifetime.

From the observed dependence of STE lifetime on the bridging-halogen ion, it is supposed that the barrier becomes lower with decrease of the CT gap energy. An experimental data which support this speculation is that the chargedsoliton pairs are efficiently produced by photoexcitation in narrow $\mathrm{CT}$ gap Pt- $X$ systems. $^{23} \mathrm{In} \mathrm{Pt}-\mathrm{Cl}$ and $\mathrm{Pt}-\mathrm{Br}$, the system is temporarily thermalized around the local potential minimum of STE due to the relatively higher energy barrier (process A in Fig. 11). Then, it goes toward the soliton-pair state by thermal activation (process B). In Pt-I, on the other hand, the system seems to go toward the soliton-pair state before it thermalizes within the STE configuration (process B') because the STE lifetime in Pt-I is extremely short (650 fs) and the time evolution of luminescence depends scarcely on the emission energy (Fig. 7). This lifetime is as short as twice the oscillation period in the STE state which is recently reported to be $270 \mathrm{fs}$ (122 $\mathrm{cm}^{-1}$ in frequency). ${ }^{20}$ This qualitatively different dynamics should be caused by the relatively lower energy barrier.

\section{SUMMARY}

The ultrafast relaxation dynamics of photoexcited CT excitons in typical 1D strongly coupled electron-lattice systems, quasi-1D halogen-bridged Pt complexes has been comprehensively studied by femtosecond time-resolved luminescence spectroscopy. In $\mathrm{Pt}-\mathrm{Br}$, the frequency spectrum of bulk phonons which compose the interaction mode of the self-trapping process is estimated by applying a calculation model to the previous results. From the spectrum, the phonon modes which contribute to triggering the localization of the electronic wave function and stabilization of the localized state are discussed. In $\mathrm{Pt}-\mathrm{Cl}$, new short-lived hot luminescence is found in the low-energy side of the STE luminescence band. The overall behavior of the STE luminescence within 2 ps including the hot luminescence is well reproduced by the same model with assuming an appropriate phonon spectral density function, and is understood by the vibrational relaxation of the STE. In Pt-I, it is found that the STE has much shorter lifetime than in $\mathrm{Pt}-\mathrm{Cl}$ and $\mathrm{Pt}-\mathrm{Br}$. By comparing the lifetimes in the three $\mathrm{Pt}-X$ systems at room temperature, it is found that the lifetime becomes shorter with decrease of the CT gap energy. This is attributable to the lowering of the energy barrier height in the nonradiative annihilation path.

\section{ACKNOWLEDGMENTS}

The authors thank Professor Y. Kayanuma and Professor S. Tanaka (Osaka Prefecture University) for stimulative discussions. This work has been supported by Grant-in-Aid for Scientific Research (A), (B) and Grant-in-Aid on priority areas, "Photo-induced Phase Transition and Their Dynamics," from the Ministry of Education, Culture, Sports, Science, and Technology of Japan.
*Present address: Correlated Electron Research Center (CERC), National Institute of Advanced Industrial Science and Technology (AIST), Tsukuba, Ibaraki 305-8562, Japan; Electronic address: s-tomimoto@aist.go.jp

${ }^{1}$ K. S. Song and R. T. Williams, Self-Trapped Excitons, Vol. 105 of Solid-State Sciences (Springer, Berlin, 1993).
${ }^{2}$ Y. Wada, T. Mitani, M. Yamashita, and T. Koda, J. Phys. Soc. Jpn. 54, 3143 (1985).

${ }^{3}$ S.C. Huckett, B. Scott, S.P. Love, R.J. Donohoe, C.J. Burns, E. Garcia, T. Frankcom, and B.I. Swanson, Inorg. Chem. 32, 2137 (1993).

${ }^{4}$ H. Endres, H.J. Keller, R. Martin, H.N. Gung, and U. Traeger, 
Acta Crystallogr., Sect. B: Struct. Crystallogr. Cryst. Chem. 35, 1885 (1979).

${ }^{5}$ J. Takeda, M. Okada, S. Kurita, K. Tanaka, and T. Suemoto, Phys. Rev. B 52, 14441 (1995).

${ }^{6}$ R.J. Donohoe, L.A. Worl, C.A. Arrington, A. Bulou, and B.I. Swanson, Phys. Rev. B 45, 13185 (1992).

${ }^{7}$ G.F. Strouse, B. Scott, M. Berkey, S. Johnson, T. Darling, A. Migliori, and B.I. Swanson, Synth. Met. 86, 1919 (1997).

${ }^{8}$ D. Emin and T. Holstein, Phys. Rev. Lett. 36, 323 (1976).

${ }^{9}$ M. Tanaka, S. Kurita, Y. Okada, T. Kojima, and Y. Yamada, Chem. Phys. 96, 343 (1985).

${ }^{10}$ S. Tomimoto, S. Saito, T. Suemoto, K. Sakata, J. Takeda, and S. Kurita, Phys. Rev. B 60, 7961 (1999).

${ }^{11}$ Y. Kayanuma, J. Phys. Soc. Jpn. 57, 292 (1988).

${ }^{12}$ S. Tomimoto, H. Nansei, S. Saito, T. Suemoto, J. Takeda, and S. Kurita, Phys. Rev. Lett. 81, 417 (1998).

${ }^{13} \mathrm{H}$. Okamoto (private communication).

${ }^{14}$ S. Tanaka and Y. Kayanuma, J. Lumin. 87-89, 936 (2000).
${ }^{15}$ A. Bulou, R.J. Donohoe, and B.I. Swanson, J. Phys.: Condens. Matter 3, 1709 (1991).

${ }^{16}$ L. Degiorgi, P. Wachter, M. Haruki, and S. Kurita, Phys. Rev. B 40, 3285 (1989).

${ }^{17}$ L. Degiorgi, P. Wachter, M. Haruki, and S. Kurita, Phys. Rev. B 42, 4341 (1990).

${ }^{18}$ S.P. Love, S.C. Huckett, L.A. Worl, T.M. Frankcom, S.A. Ekberg, and B.I. Swanson, Phys. Rev. B 47, 11107 (1993).

${ }^{19}$ S.L. Dexheimer, A.D.V. Pelt, J.A. Brozik, and B.I. Swanson, Phys. Rev. Lett. 84, 4425 (2000).

${ }^{20}$ A. Sugita, T. Saito, H. Kano, M. Yamashita, and T. Kobayashi, Phys. Rev. Lett. 86, 2158 (2001).

${ }^{21}$ Y. Wada, K. Era, and M. Yamashita, Solid State Commun. 67, 953 (1988).

${ }^{22}$ Y. Wada, U. Lemmer, E.O. Göbel, M. Yamashita, and K. Toriumi, Phys. Rev. B 52, 8276 (1995).

${ }^{23}$ H. Okamoto and M. Yamashita, Bull. Chem. Soc. Jpn. 71, 2023 (1998).

${ }^{24}$ K. Iwano, J. Phys. Soc. Jpn. 66, 1088 (1997). 